\title{
Effect of Ambient Air Pollution on Hospital Readmissions among the Pediatric Asthma Patient Population in South Texas: A Case-Crossover Study
}

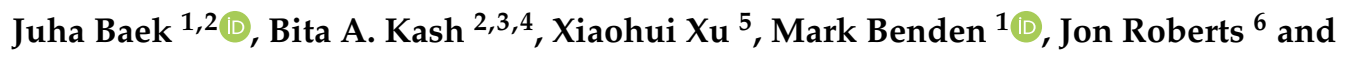 \\ Genny Carrillo $1, *$ (D) \\ 1 Department of Environmental and Occupational Health, School of Public Health, Texas A\&M University, \\ College Station, TX 77843, USA; jbaek@tamu.edu (J.B.); mthowdy@tamu.edu (M.B.) \\ 2 Center for Outcomes Research, Houston Methodist Research Institute, Houston, TX 77030, USA; \\ bakash@tamu.edu \\ 3 Center for Health \& Nature, Houston Methodist Research Institute, Houston, TX 77030, USA \\ 4 Department of Health Policy and Management, School of Public Health, Texas A\&M University, \\ College Station, TX 77843, USA \\ 5 Department of Epidemiology and Biostatistics, School of Public Health, Texas A\&M University, \\ College Station, TX 77843, USA; xiaohui.xu@tamu.edu \\ 6 Department of Pediatric Pulmonology, Driscoll Children's Hospital, Corpus Christi, TX 78411, USA; \\ Jon.Roberts@dchstx.org \\ * Correspondence: gcarrillo@tamu.edu
}

Received: 13 June 2020; Accepted: 29 June 2020; Published: 6 July 2020

\begin{abstract}
Few studies have evaluated the association between ambient air pollution and hospital readmissions among children with asthma, especially in low-income communities. This study examined the short-term effects of ambient air pollutants on hospital readmissions for pediatric asthma in South Texas. A time-stratified case-crossover study was conducted using the hospitalization data from a children's hospital and the air pollution data, including particulate matter $2.5\left(\mathrm{PM}_{2.5}\right)$ and ozone concentrations, from the Centers for Disease Control and Prevention between 2010 and 2014. A conditional logistic regression analysis was performed to investigate the association between ambient air pollution and hospital readmissions, controlling for outdoor temperature. We identified 111 pediatric asthma patients readmitted to the hospital between 2010 and 2014 . The single-pollutant models showed that $\mathrm{PM}_{2.5}$ concentration had a significant positive effect on risk for hospital readmissions ( $\mathrm{OR}=1.082,95 \% \mathrm{CI}=1.008-1.162, p=0.030)$. In the two-pollutant models, the increased risk of pediatric readmissions for asthma was significantly associated with both elevated ozone $(\mathrm{OR}=1.023,95 \% \mathrm{CI}=1.001-1.045, p=0.042)$ and $\mathrm{PM}_{2.5}$ concentrations $(\mathrm{OR}=1.080$, $95 \% \mathrm{CI}=1.005-1.161, p=0.036)$. The effects of ambient air pollutants on hospital readmissions varied by age and season. Our findings suggest that short-term (4 days) exposure to air pollutants might increase the risk of preventable hospital readmissions for pediatric asthma patients.
\end{abstract}

Keywords: hospital readmissions; pediatric asthma; ambient air pollution; $\mathrm{PM}_{2.5}$; ozone; South Texas; low-income communities

\section{Introduction}

Hospital readmission is an important asthma-related health outcome to consider, since repeated hospitalizations could lead to a large burden on patients/caregivers, hospitals, and the government with regard to health care resources, cost and quality [1,2]. In addition, a study found that children who are rehospitalized for asthma might differ in disease severity, access to care, or environmental exposures 
when compared with those who were hospitalized only once [3]. However, asthma readmissions can be potentially prevented with appropriate and timely primary care given that asthma is regarded as one of the ambulatory care-sensitive conditions [4-6]. As such, reducing preventable hospital readmissions is a key priority in order to decrease health care costs and improve quality of care and patient experience [7].

Exposure to air pollution has been regarded as a significant trigger for asthma exacerbation that may lead to repeated hospitalizations. Studies have classified air pollution into two types: indoor and outdoor. With regard to indoor air pollution, common air pollutants include moisture, smoking, dust, and chemicals - these can all serve as asthma triggers in children. Several studies found that exposure to indoor environmental conditions, such as dust mites, moisture and mold, was a significant factor for pediatric asthma exacerbations in the home setting [8,9].

Further, two studies specifically examined the relationship between indoor air quality and hospital readmissions for children with asthma. One study showed that exposure to tobacco in the indoor setting, such as detectable serum or salivary cotinine collected by biomarkers, was revealed to be significantly associated with increased risk of hospital readmission [10]. The second study found that detection of poor air quality, such as higher levels of fungi and yeast, in a child's bedroom increased the risk of hospital readmission [11]. The study also reported that having a carpeted floor in the bedroom as well as a high frequency of vacuuming at home were both significantly related with increased chance of rehospitalization for children with asthma [11].

In the case of outdoor air pollution, this subject has been studied and considered as a significant factor leading to hospital readmission among children with asthma. Previous studies have shown that exposure to traffic-related air pollution (TRAP) or residential proximity to major roads was associated with adverse respiratory health effects in children, including exacerbation of asthma symptoms [12-14]. Other studies have also examined the relationship between TRAP exposure and hospital readmission for children with asthma or bronchodilator-responsive wheezing [15-17]. In particular, one study found that higher TRAP exposure was significantly associated with greater risk of readmission in the unadjusted model, but this relationship was not significant in the adjusted model [15].

However, there is a paucity of studies that evaluate the impacts of short-term exposure to specific ambient air pollutants other than TRAP on preventable hospital readmissions among pediatric asthma patients. This lack of understanding is especially true for relatively low-income communities. To address this gap in the literature, this study examined the effects of ambient air pollutants, including $\mathrm{PM}_{2.5}$ and ozone concentrations, on the risk of preventable hospital readmission for pediatric asthma patients in South Texas.

\section{Materials and Methods}

\subsection{Data Sources and Study Setting}

Hospitalization data for pediatric asthma between 1 January 2010 and 31 December 2014 were collected from Driscoll Children's Hospital database. The hospital, which focuses on pediatric care, is located in the city of Corpus Christi, and has 189 beds serving the children of South Texas. The information recorded on the data included basic demographics (gender, age, ethnicity), type of insurance, dates of admission and discharge, the International Classification of Diseases 9th Revision (ICD-9) diagnosis code, and census tract information of each patient's residence. The inclusion criteria of the study participants were children aged 5 to 18 years old and those who were readmitted to the hospital due to asthma as a primary diagnosis (ICD-9, 493) during the study period. The subsequent admissions after index hospitalization for each patient were included as readmission cases in this study. This study protocol was reviewed and approved by the Institutional Review Boards of the Texas A\&M University (IRB2018-0857D) and Driscoll Children's Hospital.

Data on ambient air pollutants during the study period were gathered from the Centers for Disease Control and Prevention (CDC) environmental public health tracking network [18]. The daily 
average predicted atmospheric particulate matter 2.5 (or $\mathrm{PM}_{2.5}$ ) and ozone concentrations in the census tract level were estimated from the Downscaler model of the United States Environmental Protection Agency (EPA) [19]. The data used in this study were the average daily air pollution concentration, including $\mathrm{PM}_{2.5}$ and ozone, given the data availability. The data for $\mathrm{PM}_{2.5}$ and ozone indicate the mean estimated 24 hour average concentration $\left(\mu \mathrm{g} / \mathrm{m}^{3}\right)$ and the mean estimated 8 hour average concentration within three meters of the surface of the earth (parts per billion [ppb]), respectively [20]. The meteorological data of daily mean temperatures were collected from the Texas Commission on Environmental Quality (TCEQ) in order to adjust for the impact of temperature on the association between ambient air pollution and pediatric asthma rehospitalization. The geographic information system (GIS) program (ArcMap 10.4, ESRI, Redlands, CA) was used to obtain the temperature data measured in the nearest air monitoring station from each patient's residence.

\subsection{Study Design and Measurement}

A time-stratified case-crossover study design was applied to evaluate the short-term effects of ambient air pollution on hospital readmission for children with asthma. In this study, air pollution data for each patient were collected for the case period, which indicates the week of the readmission date, and three control periods, which refer to the week before, the week after, and two weeks after the readmission date. Namely, each of two air pollutant concentrations ( $\mathrm{PM}_{2.5}$ and ozone) was collected from readmission day (Lag0) to three days before readmission (Lag1-Lag3) in the week of hospital readmission for the case period and the corresponding days (4 days: Lag0-Lag3) in each of the three weeks for control periods.

To assess the relationships between ambient air pollutants and hospital readmission, we fit the models with different lag structures between the readmission day (Lag0) and three single-day lags (Lag1-Lag3). We also evaluated the associations with cumulative-day lags, indicating 2 day (Lag0-1), 3 day (Lag0-2), and 4 day (Lag0-3) moving averages of $\mathrm{PM}_{2.5}$ and ozone concentrations, since single-day lag models might underestimate the relationships [21]. For example, Lag0-2 would refer to the averaged values of each air pollutant in a total of 3 days from Lag0 to Lag2 for the case period and control periods. The air pollution exposure during the case period was compared with that of the control periods for each participant.

In addition to single-pollutant models, two-pollutant models were conducted to investigate the effect of each air pollutant on hospital readmission, controlling for the other air pollutant. Potential confounders such as individual-level characteristics were controlled by the study design, since case and control periods are compared for the same patient [22]. However, temperature information was included as a time-varying factor in the analysis.

\subsection{Statistical Analysis}

Demographic characteristics of the study population were calculated to estimate the mean and standard deviation (SD) for continuous variables and percentages for categorical variables. Pearson correlation tests were performed to assess whether ambient air pollutants ( $\mathrm{PM}_{2.5}$ and ozone) and temperature are highly correlated. A conditional logistic regression analysis was conducted to examine associations between ambient air pollution and the odds of a hospital readmission for pediatric asthma. We controlled temperature for the same periods as air pollution in all of the models. The results are presented as adjusted odds ratios (ORs) and 95\% confidence intervals (CIs). Stratified analyses by age (5-11 years old or 12-18 years old), gender (girls or boys) and season (warm: May-October or cold: November-April) were used to assess effects as a modifier. All analyses were conducted by using the Stata 14 version (StataCorp LLC, College Station, TX). All statistical tests were two sided, and a $p$-value $<0.05$ was considered to be statistically significant. 


\section{Results}

A total of 111 patients were readmitted to the children's hospital due to asthma between 2010 and 2014 (Table 1). The average age was approximately 9 years old and the number of males was higher than females (57.6 vs. $42.3 \%$ ). Most of the patients readmitted to the hospital were Hispanic (79.3\%) and approximately 70\% had public insurance (Medicaid). The average time to readmission was approximately 386 days, only $8.1 \%$ of patients had 30 day readmission and approximately $37 \%$ were readmitted to the hospital in 1 year or longer. The readmissions in the cold season were a little higher than those in the warm season (52.3\% vs. $47.7 \%)$.

Table 1. Descriptive characteristics of asthma pediatric patients readmitted to the hospital between 2010 and $2014(N=111)$.

\begin{tabular}{|c|c|}
\hline Variable & Mean \pm SD $[$ Min, Max] or $N(\%)$ \\
\hline Age (years) & $9.54 \pm 3.34[5,18]$ \\
\hline \multicolumn{2}{|l|}{ Age } \\
\hline $5-11$ years old & $88(79.3)$ \\
\hline $12-18$ years old & $23(20.7)$ \\
\hline \multicolumn{2}{|l|}{ Gender } \\
\hline Female & $47(42.3)$ \\
\hline Male & $64(57.6)$ \\
\hline \multicolumn{2}{|l|}{ Ethnicity } \\
\hline Hispanic & $88(79.3)$ \\
\hline Non-Hispanic & $23(20.7)$ \\
\hline \multicolumn{2}{|l|}{ Type of insurance } \\
\hline Public (Medicaid) & $78(70.3)$ \\
\hline Private & $29(26.1)$ \\
\hline Self-pay & $4(3.6)$ \\
\hline $\begin{array}{c}\text { Days to readmission } \\
\text { (days) }\end{array}$ & $386.5 \pm 364.24[1,1651]$ \\
\hline 1-30 days & $9(8.1)$ \\
\hline 31-90 days & $12(10.8)$ \\
\hline 91-180 days & $19(17.1)$ \\
\hline 181-365 days & $30(27.1)$ \\
\hline 366 days or longer & $41(36.9)$ \\
\hline \multicolumn{2}{|l|}{ Season } \\
\hline Warm (May-October) & $53(47.7)$ \\
\hline Cold (November-April) & $58(52.3)$ \\
\hline \multicolumn{2}{|l|}{ Year } \\
\hline 2010 & $13(11.7)$ \\
\hline 2011 & $22(19.8)$ \\
\hline 2012 & $18(16.2)$ \\
\hline 2013 & $26(23.4)$ \\
\hline 2014 & $32(28.8)$ \\
\hline
\end{tabular}

Note: SD—standard deviation.

Table 2 displays summary statistics of daily ambient air pollutant concentrations and daily temperature in South Texas between 2010 and 2014. The overall mean of $\mathrm{PM}_{2.5}$ and ozone concentrations was $8.3\left(\mu \mathrm{g} / \mathrm{m}^{3}\right)$ and $37.37(\mathrm{ppb})$, respectively. The overall average temperature in the study region was $20.2^{\circ} \mathrm{C}$ and its range was between 8.83 and $29.73^{\circ} \mathrm{C}$. The results of the Pearson correlation tests showed that ambient air pollutant and temperature were not highly correlated with each other (correlation coefficient $\mathrm{r}=-0.214$ to $0.077, p<0.05$ ) (See Appendix A Table A1). 
Table 2. Summary statistics of daily ambient air pollutant concentrations and daily temperature in South Texas between 2010 and 2014.

\begin{tabular}{ccccccccc}
\hline \multirow{2}{*}{ Variable } & \multirow{2}{*}{ Mean } & \multirow{2}{*}{ SD } & \multirow{2}{*}{ Minimum } & \multicolumn{3}{c}{ Percentile } & \multirow{2}{*}{ Maximum } & \multirow{2}{*}{ IQR } \\
\cline { 5 - 7 } & & & & $\mathbf{2 5}$ & $\mathbf{5 0}$ & $\mathbf{7 5}$ & & \\
\hline $\mathrm{PM}_{2.5}\left(\mu \mathrm{g} / \mathrm{m}^{3}\right)$ & 8.30 & 1.49 & 4.66 & 7.19 & 8.25 & 9.19 & 12.37 & 2.0 \\
Ozone $(\mathrm{ppb})$ & 37.37 & 6.81 & 20.53 & 31.47 & 37.24 & 42.11 & 52.84 & 10.64 \\
Temperature $\left({ }^{\circ} \mathrm{C}\right)$ & 20.20 & 5.85 & 8.83 & 16.31 & 21.01 & 26.91 & 29.73 & 10.6 \\
\hline
\end{tabular}

Note: SD—standard deviation; IQR—interquartile ranges.

Table 3 describes the results of the conditional logistic regression analysis to examine the association between ambient air pollution and preventable hospital readmission. We found that the elevated $\mathrm{PM}_{2.5}$ concentration was significantly associated with an increased risk of preventable hospital readmission on Lag1 in both single-pollutant $(\mathrm{OR}=1.082,95 \% \mathrm{CI}=1.008-1.162, p=0.030)$ and two-pollutant models ( $\mathrm{OR}=1.080,95 \% \mathrm{CI}=1.005-1.161, p=0.036)$, controlling for temperature. Further, we observed a significant positive association of preventable hospital readmission with ozone concentration on $\mathrm{Lag} 0$ in the two-pollutant model (OR $=1.023,95 \% \mathrm{CI}=1.001-1.045, p=0.042)$. However, none of the ambient air pollutants were significant on cumulative-day lags (Lag0-1 Lag0-3) in the single- and two-pollutant models. Figure 1 illustrates the lag structures of ORs and 95\% CIs of $\mathrm{PM}_{2.5}$ and ozone concentrations with preventable hospital readmission for the patients on single- and multiple-day lags in the single- and two-pollutant models.

Table 3. Results of the conditional logistic regression models between ambient air pollution and hospital readmission.

\begin{tabular}{|c|c|c|c|c|c|c|c|c|}
\hline \multirow{3}{*}{$\begin{array}{l}\text { Single- and } \\
\text { Cumulative- } \\
\text { Day Lags }\end{array}$} & \multicolumn{4}{|c|}{ Single-Pollutant Model } & \multicolumn{4}{|c|}{ Two-Pollutant Model } \\
\hline & \multicolumn{2}{|c|}{$\mathbf{P M}_{2.5}$} & \multicolumn{2}{|c|}{ Ozone } & \multicolumn{2}{|c|}{$\mathbf{P M}_{2.5}$} & \multicolumn{2}{|c|}{ Ozone } \\
\hline & OR $(95 \% \mathrm{CI})$ & $p$-Value & OR $(95 \% \mathrm{CI})$ & $p$-Value & OR $(95 \% \mathrm{CI})$ & $p$-Value & OR $(95 \% \mathrm{CI})$ & $p$-Value \\
\hline Lag0 & $\begin{array}{c}0.972 \\
(0.897-1.052)\end{array}$ & 0.479 & $\begin{array}{c}1.020 \\
(0.999-1.041)\end{array}$ & 0.064 & $\begin{array}{c}0.954 \\
(0.878-1.037)\end{array}$ & 0.272 & $\begin{array}{c}1.023 \\
(1.001-1.045)\end{array}$ & $0.042 *$ \\
\hline Lag1 & $\begin{array}{c}1.082 \\
(1.008-1.162)\end{array}$ & $0.030 *$ & $\begin{array}{c}1.008 \\
(0.987-1.028)\end{array}$ & 0.469 & $\begin{array}{c}1.080 \\
(1.005-1.161)\end{array}$ & $0.036^{*}$ & $\begin{array}{c}1.005 \\
(0.984-1.025)\end{array}$ & 0.665 \\
\hline Lag2 & $\begin{array}{c}0.984 \\
(0.914-1.059)\end{array}$ & 0.664 & $\begin{array}{c}1.001 \\
(0.979-1.022)\end{array}$ & 0.955 & $\begin{array}{c}0.983 \\
(0.912-1.059)\end{array}$ & 0.655 & $\begin{array}{c}1.001 \\
(0.980-1.023)\end{array}$ & 0.904 \\
\hline Lag3 & $\begin{array}{c}0.985 \\
(0.914-1.063)\end{array}$ & 0.700 & $\begin{array}{c}1.007 \\
(0.988-1.027)\end{array}$ & 0.472 & $\begin{array}{c}0.980 \\
(0.907-1.059)\end{array}$ & 0.613 & $\begin{array}{c}1.008 \\
(0.988-1.028)\end{array}$ & 0.429 \\
\hline Lag0-1 & $\begin{array}{c}1.046 \\
(0.954-1.147)\end{array}$ & 0.334 & $\begin{array}{c}1.016 \\
(0.993-1.039)\end{array}$ & 0.168 & $\begin{array}{c}1.036 \\
(0.942-1.138)\end{array}$ & 0.468 & $\begin{array}{c}1.014 \\
(0.992-1.037)\end{array}$ & 0.222 \\
\hline Lag0-2 & $\begin{array}{c}1.032 \\
(0.929-1.145)\end{array}$ & 0.558 & $\begin{array}{c}1.013 \\
(0.989-1.038)\end{array}$ & 0.292 & $\begin{array}{c}1.023 \\
(0.921-1.138)\end{array}$ & 0.667 & $\begin{array}{c}1.012 \\
(0.988-1.037)\end{array}$ & 0.667 \\
\hline Lag0-3 & $\begin{array}{c}1.017 \\
(0.907-1.140)\end{array}$ & 0.779 & $\begin{array}{c}1.015 \\
(0.988-1.042)\end{array}$ & 0.275 & $\begin{array}{c}1.006 \\
(0.896-1.131)\end{array}$ & 0.915 & $\begin{array}{c}1.015 \\
(0.988-1.042)\end{array}$ & 0.290 \\
\hline
\end{tabular}

Note: Models adjusted for temperature; ${ }^{*} p<0.05$.

Table 4 demonstrates the results of age-stratified conditional logistic regression models. We found that the association between ozone concentration and risk of preventable readmission was significantly positive on Lag0 in the two-pollutant model among children aged 5-11 years, adjusting for temperature $(\mathrm{OR}=1.029,95 \% \mathrm{CI}=1.004-1.055, p=0.022)$. Yet, none of the associations for $\mathrm{PM}_{2.5}$ concentration were significant. On the other hand, there was no significant relationship of ambient air pollutants with preventable hospital readmission among those aged 12-18 years old. The gender-stratified models shown in Table 5 show that associations between ambient air pollutants and pediatric asthma rehospitalization were not significant among males and females. 


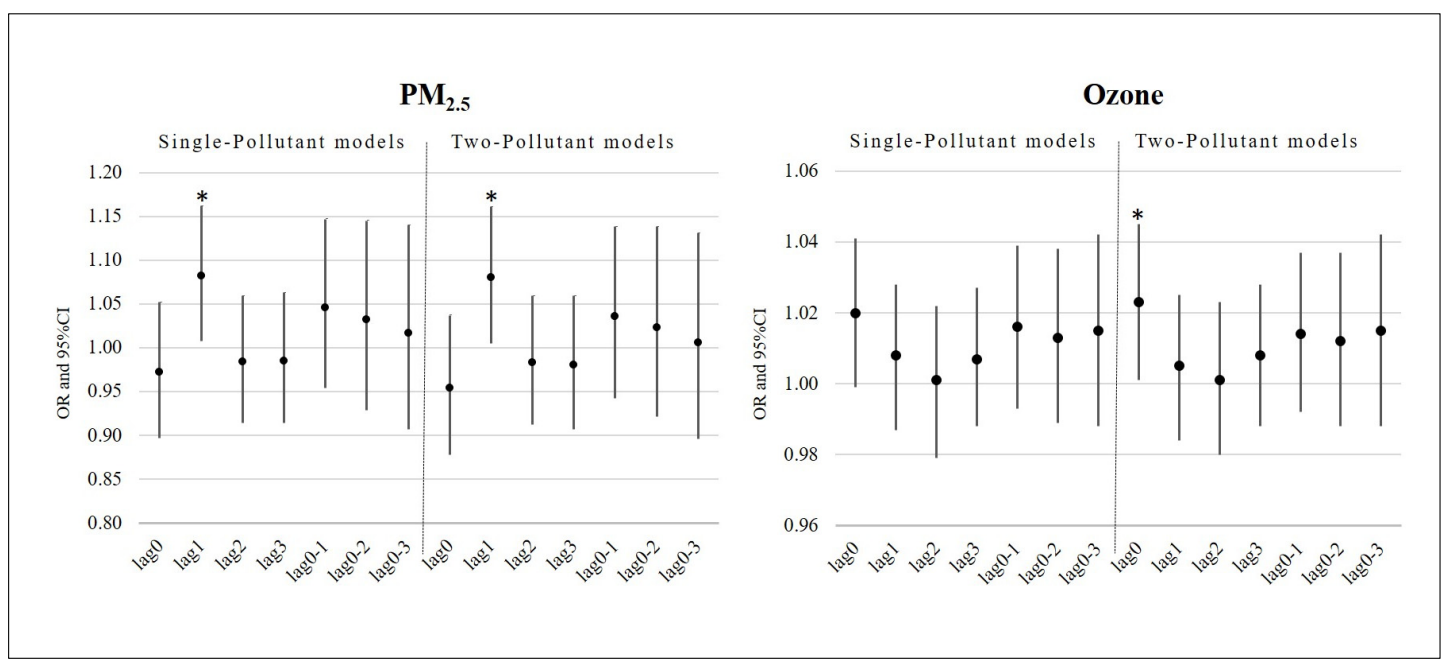

Figure 1. Odds ratio (OR) and 95\% confidence interval (CI) of $\mathrm{PM}_{2.5}$ and ozone with hospital readmissions for children with asthma in the single- and two-pollutant models $\left({ }^{*} p<0.05\right)$.

Table 4. Results of the conditional logistic regression models stratified by age.

\begin{tabular}{|c|c|c|c|c|c|c|c|c|}
\hline \multirow{3}{*}{$\begin{array}{l}\text { Single- and } \\
\text { Cumulative- } \\
\text { Day Lags }\end{array}$} & \multicolumn{4}{|c|}{ 5-11 Years Old } & \multicolumn{4}{|c|}{ 12-18 Years Old } \\
\hline & \multicolumn{2}{|c|}{ Single-Pollutant } & \multicolumn{2}{|c|}{ Two-Pollutant } & \multicolumn{2}{|c|}{ Single-Pollutant } & \multicolumn{2}{|c|}{ Two-Pollutant } \\
\hline & $\mathrm{PM}_{2.5}$ & Ozone & $\mathbf{P M}_{2.5}$ & Ozone & $\mathrm{PM}_{2.5}$ & Ozone & $\mathbf{P M}_{2.5}$ & Ozone \\
\hline Lag0 & $\begin{array}{c}0.934 \\
(0.850-1.026)\end{array}$ & $\begin{array}{c}1.022 \\
(0.999-1.047)\end{array}$ & $\begin{array}{c}0.908 \\
(0.821-1.004)\end{array}$ & $\begin{array}{c}1.029^{*} \\
(1.004-1.055)\end{array}$ & $\begin{array}{c}1.123 \\
(0.942-1.337)\end{array}$ & $\begin{array}{c}1.009 \\
(0.964-1.057)\end{array}$ & $\begin{array}{c}1.122 \\
(0.942-1.336)\end{array}$ & $\begin{array}{c}1.008 \\
(0.965-1.054)\end{array}$ \\
\hline Lag1 & $\begin{array}{c}1.075 \\
(0.994-1.162)\end{array}$ & $\begin{array}{c}1.009 \\
(0.986-1.033)\end{array}$ & $\begin{array}{c}1.071 \\
(0.990-1.159)\end{array}$ & $\begin{array}{c}1.006 \\
(0.982-1.030)\end{array}$ & $\begin{array}{c}1.112 \\
(0.924-1.338)\end{array}$ & $\begin{array}{c}1.001 \\
(0.959-1.046)\end{array}$ & $\begin{array}{c}1.113 \\
(0.924-1.339)\end{array}$ & $\begin{array}{c}1.002 \\
(0.960-1.045)\end{array}$ \\
\hline Lag2 & $\begin{array}{c}0.962 \\
(0.881-1.050)\end{array}$ & $\begin{array}{c}1.003 \\
(0.980-1.027)\end{array}$ & $\begin{array}{c}0.959 \\
(0.878-1.048)\end{array}$ & $\begin{array}{c}1.005 \\
(0.981-1.029)\end{array}$ & $\begin{array}{c}1.045 \\
(0.908-1.204)\end{array}$ & $\begin{array}{c}0.983 \\
(0.933-1.034)\end{array}$ & $\begin{array}{c}1.062 \\
(0.917-1.230)\end{array}$ & $\begin{array}{c}0.978 \\
(0.927-1.031)\end{array}$ \\
\hline Lag3 & $\begin{array}{c}0.983 \\
(0.905-1.067)\end{array}$ & $\begin{array}{c}1.004 \\
(0.982-1.026)\end{array}$ & $\begin{array}{c}0.980 \\
(0.902-1.066)\end{array}$ & $\begin{array}{c}1.005 \\
(0.983-1.027)\end{array}$ & $\begin{array}{c}0.969 \\
(0.795-1.182)\end{array}$ & $\begin{array}{c}1.016 \\
(0.968-1.067)\end{array}$ & $\begin{array}{c}0.953 \\
(0.779-1.166)\end{array}$ & $\begin{array}{c}1.019 \\
(0.969-1.071)\end{array}$ \\
\hline Lag0-1 & $\begin{array}{c}1.017 \\
(0.917-1.127)\end{array}$ & $\begin{array}{c}1.018 \\
(0.993-1.044)\end{array}$ & $\begin{array}{c}1.000 \\
(0.899-1.112)\end{array}$ & $\begin{array}{c}1.018 \\
(0.992-1.045)\end{array}$ & $\begin{array}{c}1.174 \\
(0.945-1.459)\end{array}$ & $\begin{array}{c}1.006 \\
(0.958-1.056)\end{array}$ & $\begin{array}{c}1.176 \\
(0.946-1.462)\end{array}$ & $\begin{array}{c}1.007 \\
(0.960-1.055)\end{array}$ \\
\hline Lag0-2 & $\begin{array}{c}0.994 \\
(0.880-1.122)\end{array}$ & $\begin{array}{c}1.016 \\
(0.989-1.044)\end{array}$ & $\begin{array}{c}0.982 \\
(0.868-1.111)\end{array}$ & $\begin{array}{c}1.017 \\
(0.989-1.045)\end{array}$ & $\begin{array}{c}1.152 \\
(0.923-1.437)\end{array}$ & $\begin{array}{c}1.000 \\
(0.948-1.056)\end{array}$ & $\begin{array}{c}1.153 \\
(0.923-1.440)\end{array}$ & $\begin{array}{c}0.997 \\
(0.946-1.051)\end{array}$ \\
\hline Lag0-3 & $\begin{array}{c}0.983 \\
(0.861-1.121)\end{array}$ & $\begin{array}{c}1.016 \\
(0.987-1.047)\end{array}$ & $\begin{array}{c}0.970 \\
(0.848-1.110)\end{array}$ & $\begin{array}{c}1.017 \\
(0.987-1.048)\end{array}$ & $\begin{array}{c}1.123 \\
(0.879-1.435)\end{array}$ & $\begin{array}{c}1.006 \\
(0.948-1.067)\end{array}$ & $\begin{array}{c}1.122 \\
(0.877-1.435)\end{array}$ & $\begin{array}{c}1.003 \\
(0.946-1.063)\end{array}$ \\
\hline
\end{tabular}

Note: Models adjusted for temperature; ${ }^{*} p<0.05$.

Table 5. Results of the conditional logistic regression analysis stratified by gender.

\begin{tabular}{|c|c|c|c|c|c|c|c|c|}
\hline \multirow{3}{*}{$\begin{array}{l}\text { Single- and } \\
\text { Cumulative- } \\
\text { Day Lags }\end{array}$} & \multicolumn{4}{|c|}{ Girls } & \multicolumn{4}{|c|}{ Boys } \\
\hline & \multicolumn{2}{|c|}{ Single-Pollutant } & \multicolumn{2}{|c|}{ Two-Pollutant } & \multicolumn{2}{|c|}{ Single-Pollutant } & \multicolumn{2}{|c|}{ Two-Pollutant } \\
\hline & $\mathrm{PM}_{2.5}$ & Ozone & $\mathbf{P M}_{2.5}$ & Ozone & $\mathrm{PM}_{2.5}$ & Ozone & $\mathrm{PM}_{2.5}$ & Ozone \\
\hline \multirow{2}{*}{ Lag0 } & 0.936 & 1.021 & 0.902 & 1.029 & 0.994 & 1.019 & 0.983 & 1.019 \\
\hline & $(0.819-1.070)$ & $(0.989-1.054)$ & $(0.776-1.048)$ & $(0.993-1.066)$ & (0.900-1.099) & (0.991-1.047) & $(0.888-1.089)$ & (0.991-1.048) \\
\hline \multirow{2}{*}{ Lag1 } & 1.113 & 1.012 & 1.108 & 1.007 & 1.061 & 1.002 & 1.061 & 1.001 \\
\hline & $(0.997-1.244)$ & $(0.984-1.042)$ & $(0.989-1.240)$ & $(0.977-1.037)$ & $(0.965-1.166)$ & $(0.973-1.032)$ & $(0.965-1.166)$ & $(0.972-1.031)$ \\
\hline \multirow{2}{*}{ Lag2 } & 0.972 & 1.003 & 0.967 & 1.006 & 0.986 & 0.996 & 0.986 & 0.996 \\
\hline & $(0.858-1.102)$ & $(0.973-1.035)$ & $(0.849-1.101)$ & $(0.974-1.038)$ & $(0.900-1.079)$ & (0.967-1.027) & $(0.900-1.080)$ & $(0.967-1.027)$ \\
\hline \multirow{2}{*}{ Lag3 } & 0.984 & 1.007 & 0.972 & 1.010 & 0.985 & 1.005 & 0.984 & 1.006 \\
\hline & $(0.875-1.107)$ & (0.979-1.037) & $(0.858-1.100)$ & $(0.980-1.041)$ & $(0.892-1.087)$ & (0.979-1.033) & (0.891-1.087) & $(0.979-1.033)$ \\
\hline \multirow{2}{*}{ Lag0-1 } & 1.053 & 1.018 & 1.032 & 1.016 & 1.044 & 1.012 & 1.038 & 1.011 \\
\hline & $(0.911-1.218)$ & (0.986-1.052) & $(0.885-1.203)$ & $(0.983-1.051)$ & $(0.926-1.175)$ & $(0.982-1.044)$ & (0.921-1.171) & $(0.980-1.043)$ \\
\hline \multirow{2}{*}{ Lag0-2 } & 1.038 & 1.016 & 1.019 & 1.015 & 1.026 & 1.009 & 1.025 & 1.008 \\
\hline & $(0.882-1.222)$ & $(0.981-1.052)$ & $(0.859-1.208)$ & $(0.979-1.052)$ & $(0.897-1.175)$ & (0.974-1.044) & (0.895-1.173) & (0.974-1.044) \\
\hline \multirow{2}{*}{ Lag0-3 } & 1.015 & 1.016 & 0.989 & 1.017 & 1.013 & 1.011 & 1.012 & 1.011 \\
\hline & (0.852-1.209) & (0.979-1.054) & (0.821-1.193) & (0.978-1.057) & $(0.872-1.176)$ & $(0.973-1.050)$ & (0.871-1.176) & $(0.973-1.050)$ \\
\hline
\end{tabular}

Note: Models adjusted for temperature.

Table 6 displays the results of the conditional logistic regression analysis stratified by season. We observed that both $\mathrm{PM}_{2.5}$ and ozone had more of an effect on the risk of preventable rehospitalization 
during the warm season (May-October) compared with the cold season (November-April). Specifically, the association between $\mathrm{PM}_{2.5}$ and preventable readmission was found to be positively significant on Lag1 in the single-pollutant $(\mathrm{OR}=1.134,95 \% \mathrm{CI}=1.019-1.262, p=0.021)$ and two-pollutant models (OR $=1.125,95 \% \mathrm{CI}=1.009-1.254, p=0.034)$ as well as on Lag0-1 in the single-pollutant model $(\mathrm{OR}=1.146,95 \% \mathrm{CI}=1.003-1.309, p=0.046)$ in the warm season. For ozone, positive relationships with hospital readmission were observed on $\mathrm{Lag} 0$ in both single-pollutant $(\mathrm{OR}=1.043,95 \% \mathrm{CI}=1.012-1.075$, $p=0.007)$ and two-pollutant models ( $\mathrm{OR}=1.043,95 \% \mathrm{CI}=1.010-1.078, p=0.01)$, and on Lag0-1 in the single-pollutant model (OR $=1.033,95 \% \mathrm{CI}=1.002-1.065, p=0.037)$ in the warm season. No significant associations were found in the cold season.

Table 6. Results of the conditional logistic regression analysis stratified by season.

\begin{tabular}{|c|c|c|c|c|c|c|c|c|}
\hline \multirow{3}{*}{$\begin{array}{l}\text { Single- and } \\
\text { Cumulative- } \\
\text { Day Lags }\end{array}$} & \multicolumn{4}{|c|}{ Warm Season (May-October) } & \multicolumn{4}{|c|}{ Cold Season (November-April) } \\
\hline & \multicolumn{2}{|c|}{ Single-Pollutant } & \multicolumn{2}{|c|}{ Two-Pollutant } & \multicolumn{2}{|c|}{ Single-Pollutant } & \multicolumn{2}{|c|}{ Two-Pollutant } \\
\hline & $\mathbf{P M}_{2.5}$ & Ozone & $\mathbf{P M}_{2.5}$ & Ozone & $\mathbf{P M}_{2.5}$ & Ozone & $\mathbf{P M}_{2.5}$ & Ozone \\
\hline \multirow{2}{*}{ Lag0 } & 1.056 & $1.043^{* *}$ & 0.993 & $1.043 *$ & 0.920 & 1.023 & 0.922 & 1.022 \\
\hline & $(0.941-1.184)$ & $(1.012-1.075)$ & $(0.874-1.127)$ & $(1.010-1.078)$ & $(0.818-1.034)$ & $(0.986-1.062)$ & $(0.818-1.038)$ & $(0.985-1.061)$ \\
\hline \multirow{2}{*}{ Lag1 } & 1.134 * & 1.016 & 1.125 * & 1.009 & 1.032 & 1.013 & 1.030 & 1.013 \\
\hline & $(1.019-1.262)$ & $(0.989-1.043)$ & $(1.009-1.254)$ & $(0.982-1.037)$ & $(0.933-1.141)$ & $(0.975-1.053)$ & $(0.931-1.138)$ & $(0.975-1.052)$ \\
\hline \multirow{2}{*}{ Lag2 } & 1.038 & 1.003 & 1.037 & 1.001 & 0.934 & 1.008 & 0.932 & 1.009 \\
\hline & (0.933-1.155) & $(0.976-1.031)$ & $(0.930-1.158)$ & $(0.973-1.030)$ & $(0.837-1.042)$ & $(0.970-1.047)$ & $(0.835-1.041)$ & $(0.970-1.050)$ \\
\hline \multirow{2}{*}{ Lag3 } & 1.011 & 1.013 & 0.995 & 1.014 & 0.956 & 1.011 & 0.956 & 1.011 \\
\hline & $(0.915-1.116)$ & (0.989-1.039) & $(0.895-1.106)$ & $(0.988-1.041)$ & $(0.853-1.071)$ & $(0.973-1.051)$ & $(0.853-1.071)$ & $(0.972-1.052)$ \\
\hline \multirow{2}{*}{ Lag0-1 } & 1.146 * & 1.033 * & 1.105 & 1.025 & 0.965 & 1.023 & 0.968 & 1.022 \\
\hline & (1.003-1.309) & $(1.002-1.065)$ & $(0.961-1.271)$ & $(0.993-1.059)$ & $(0.844-1.104)$ & $(0.982-1.066)$ & $(0.846-1.108)$ & $(0.981-1.066)$ \\
\hline \multirow{2}{*}{ Lag0-2 } & 1.151 & 1.026 & 1.123 & 1.020 & 0.926 & 1.023 & 0.927 & 1.023 \\
\hline & $(0.989-1.340)$ & $(0.994-1.060)$ & $(0.960-1.314)$ & $(0.987-1.054)$ & $(0.793-1.081)$ & $(0.977-1.071)$ & $(0.795-1.082)$ & $(0.977-1.072)$ \\
\hline \multirow{2}{*}{ Lag0-3 } & 1.132 & 1.031 & 1.098 & 1.025 & 0.892 & 1.029 & 0.894 & 1.029 \\
\hline & $(0.965-1.329)$ & (0.997-1.066) & $(0.929-1.296)$ & $(0.990-1.062)$ & $(0.744-1.070)$ & $(0.976-1.085)$ & $(0.747-1.070)$ & (0.975-1.087) \\
\hline
\end{tabular}

\section{Discussion}

This study examined the effects of ambient air pollution on preventable hospital readmission among children with asthma in South Texas. We found that short-term exposure to $\mathrm{PM}_{2.5}$ concentration was positively associated with an increased risk of preventable rehospitalization for pediatric asthma in both the single- and two-pollutant models. Ozone pollutant also had a significantly positive effect on asthma hospital readmission in the two-pollutant model. Specifically, significant associations between ambient air pollutants and hospital readmissions were found in the readmission day (Lag0) and the day before readmission day (Lag1).

The findings of this study provide support for the adverse effects of ambient air pollutants on the risk of preventable hospital readmission for pediatric asthma. This is important new knowledge, given that previous studies only included traffic-related air pollutants (TRAPs). For example, a study reported that residential exposure to specific TRAPs including $\mathrm{NO}_{x}$ and $\mathrm{CO}$ was significantly associated with an elevated risk of rehospitalizations for pediatric asthma [16]. Another study targeting children with asthma or bronchodilator-responsive wheezing showed that exposure to TRAPs affected hospital readmission, especially among white children [15]. In addition, one other study showed that the residential proximity to major roads or freeways (within 300 meters) increased the risk of hospital readmission for pediatric asthma [17]. As a result, the current study extends the findings of earlier evidence about a relationship between outdoor air pollutants and hospital readmissions for asthma.

The results of this study were consistent with those of previous studies that explored the effect of ambient air pollutants on pediatric asthma exacerbations, including hospital admission and emergency department (ED) visits. A review of 22 studies reported that exposures to $\mathrm{PM}_{2.5}$, sulfur dioxide $\left(\mathrm{SO}_{2}\right)$, and nitrogen dioxide $\left(\mathrm{NO}_{2}\right)$ were significantly associated with asthma exacerbation among children less than 18 years of age [23]. Multiple studies have also found positive relationships between $\mathrm{PM}_{2.5}$ and asthma hospitalization or ED visits for pediatric patients [24-27]. Moreover, previous research 
revealed the adverse effects of ozone concentration on asthma exacerbations for children in different settings, such as Texas [28], New York [29], California [30], China [24], HongKong [31] and Korea [32].

Based on this study, the effects of ambient air pollutants on preventable hospital readmission varied by age and season. We found that ozone concentration may contribute to increasing the odds of preventable hospital readmission for younger children aged 5-11 years but not for those aged 12-18 years. There is no evidence that substantiates the effects of $\mathrm{PM}_{2.5}$ or ozone on pediatric asthma rehospitalization by age; however, one study presented that traffic-related $\mathrm{NO}_{\mathrm{x}}$ and $\mathrm{CO}$ had a significant age effect on readmission for infants but not for children aged 1 to 18 years [16]. Age-stratified models of studies reporting the impact of $\mathrm{PM}_{2.5}$ and ozone on asthma pediatric exacerbations had mixed results in different age groups. Studies found a significant association between air pollutants $\left(\mathrm{PM}_{2.5}\right.$ and/or ozone) and asthma exacerbations among preschool children (5 years or less) [25,26,33], those aged 5-14 years $[25,34]$, and those aged $6-18$ years [24,33].

The models stratified by season showed significant positive effects of $\mathrm{PM}_{2.5}$ and ozone on preventable hospital readmission during the warm season, while such effects were not observed during the cold season. Our results were consistent with those of previous studies that examined the association between ambient air pollutants and asthma exacerbations for pediatric asthma. Most studies that were previously conducted found a significant effect of $\mathrm{PM}_{2.5}$ on asthma hospitalization or ED visits during the warm season (May-October or April-September), and none in the cold season $[25,26,35,36]$. Another study also found a stronger association in the warm season than in the cold season [34]. Additionally, other studies have also revealed a significant relationship between ozone and asthma exacerbations in the warm season $[35,37,38]$. This finding is most likely due to the fact that children tend to play outside or have more outdoor activities at home and at school during the warm season compared to the cold season. Their houses are also more likely to be ventilated during the warm season, which may increase their personal exposure to ambient air pollutants $[25,39,40]$.

The models stratified by gender did not show any significant associations in the current study. This finding is contrary to previous research, which suggested that gender could be a modifying factor of the relationship between TRAPs and hospital readmission. One study found that TRAPs including $\mathrm{NO}_{\mathrm{x}}$ and $\mathrm{CO}$ were associated with pediatric asthma rehospitalizations among females only [16]. Another study revealed that the adverse impact of residence near heavy traffic on repeated asthma hospitalization was stronger among females than males (in the 6-18 years of age group) [17]. The gender difference was also observed in previous studies that investigated the relationship between ambient air pollutants $\left(\mathrm{PM}_{2.5}\right.$ and ozone) and asthma exacerbations for pediatric asthma [24-26,33].

\subsection{Limitations}

This study has several limitations. First, we used daily average air pollutant concentrations predicted by the statistical model at the census tract level as a proxy for personal exposure to outdoor air pollution, which may cause an exposure measurement error and thus underestimate the impacts of ambient air pollutants [41]. Second, the temperature data may not be accurate, since the distance from the residence to the closest monitoring sites varied for each patient. This may also lead to measurement error. Third, only one children's hospital in South Texas, where ambient air pollutant concentrations were relatively low, was included in this study. As such, the findings may not be generalizable to other settings in different regions. Further study should be conducted in other regions with higher ambient air pollution to confirm the association. Fourth, some important factors that may potentially be associated with personal exposure to air pollution, such as the amount of time spent for outdoor activities and the level of indoor air pollution, and other air pollutants like $\mathrm{NO}_{2}$ were not included in this study due to unavailability of data. Finally, air pollution data were linked to the patients' residential census tract; however, we cannot guarantee that the patients were actually living or staying in that address during the study period. 


\subsection{Health Policy and Practice Implications}

The findings of this study have several important implications for public health and health care services to reduce preventable hospital readmission. First, it would be important for children with asthma, who have experienced hospitalization earlier, to limit outdoor activities and/or habitually wear a facemask when going outside on days with high levels of $\mathrm{PM}_{2.5}$ and ozone concentrations. This will help minimize personal exposure to air pollutants. The parents or families with younger children with asthma should also be more cautious about their children's outdoor activities on days with high levels of ambient air pollutants and particularly during the warm season. Second, the results of this study can help health care professionals, who serve pediatric patients with asthma, to understand that ambient air pollutants, including $\mathrm{PM}_{2.5}$ and ozone, could increase the risk of preventable hospital readmission among children with asthma. Third, this study's findings may help policy makers to develop a policy to control ambient air pollution in order to reduce emissions of $\mathrm{PM}_{2.5}$ and ozone.

Fourth, previous evidence showed that pre-discharge patient/family education is a successful intervention to reduce preventable readmission [42,43]. Therefore, it would be important for hospital leaders or health care workers in children's hospitals to consider emphasizing the contents related to ambient air pollution when delivering education to the children with asthma and their family before discharge as one of the readmission reduction initiatives. The educational contents may include the effects of ambient air pollution, federal standards for each air pollutant, and how to check daily outdoor air quality and prevent air pollutant exposure. Finally, children's hospitals will be able to collaborate with asthma education/prevention programs in the community to follow up with pediatric patients with asthma more effectively, given a study's argument that optimal inpatient asthma care includes an effective transition to the community with constant follow-up care to prevent repeated hospitalization [42]. Home-visit asthma education has especially been proven to be effective in improving asthma-related health outcomes for the population living in disadvantaged communities with limited access to health care and education [44-46]. The community-hospital partnership for education and follow up for pediatric patients with asthma may play a significant role in reducing preventable hospital readmission [47].

\section{Conclusions}

This is the first study, to the best of our knowledge, which investigates the association between short-term exposures to ambient air pollution and preventable hospital readmissions for pediatric asthma patients in South Texas. Our study confirms the adverse effects of $\mathrm{PM}_{2.5}$ and ozone concentrations on preventable hospital readmission among children with asthma in low-income communities. We discovered that younger age and exposure during the warmer season were associated with the effects of ambient air pollutants. Our findings contribute to the limited scientific evidence regarding the effect of ambient air pollutants on hospital readmission for pediatric asthma. However, further research is still warranted to confirm our findings.

Author Contributions: Conceptualization, J.B., B.A.K., X.X., M.B. and G.C.; data curation, J.B.; formal analysis, J.B.; investigation, J.B., J.R. and G.C.; methodology, J.B. and X.X.; visualization, J.B.; supervision, B.A.K., M.B. and G.C.; funding acquisition, J.R. and G.C.; writing-original draft, J.B.; writing-review and editing, J.B., B.A.K., X.X., M.B., J.R. and G.C.; resources, J.R. and G.C.; project administration, G.C. All authors have read and agreed to the published version of the manuscript.

Funding: This work was supported by the Global Institute for Hispanic Health, and the APC was funded by Healthy South Texas.

Acknowledgments: The authors would like to thank Erin Richmond (program manager) and Jennifer Phillips (data analyst), from Driscoll Children's Hospital, and Scott Horel (data manager) from Texas A\&M University School of Public Health, who helped with extracting and converting the data. We would also like to thank Jacob M. Kolman, the Senior Scientific Writer of the Houston Methodist Research Institute Center for Outcomes Research, for his review and editorial support.

Conflicts of Interest: The authors declare no conflict of interest. 


\section{Appendix A}

Table A1. Pearson correlation coefficients among daily concentrations of air pollutants and daily average temperature.

\begin{tabular}{cccc}
\hline Variable & $\mathbf{P M}_{2.5}$ & Ozone & Temperature \\
\hline $\mathrm{PM}_{2.5}$ & 1.00 & & \\
Ozone & $-0.214^{*}$ & 1.00 & 1.00 \\
Temperature & $-0.216^{*}$ & 0.077 & \\
\hline \multicolumn{4}{c}{ Note: significant at $p<0.05\left(^{*}\right)}$.
\end{tabular}

\section{References}

1. Watson, L.; Turk, F.; Rabe, K.F. Burden of asthma in the hospital setting: An Australian analysis. Int. J. Clin Pract. 2007, 61, 1884-1888. [CrossRef]

2. Berry, J.G.; Hall, D.E.; Kuo, D.Z.; Cohen, E.; Agrawal, R.; Feudtner, C.; Hall, M. Hospital utilization and characteristics of patients experiencing recurrent readmissions within children's hospitals. JAMA 2011, 305, 682-690. [CrossRef] [PubMed]

3. Liu, S.Y.; Pearlman, D.N. Hospital readmissions for childhood asthma: The role of individual and neighborhood factors. Public Health Rep. 2009, 124, 65-78. [CrossRef] [PubMed]

4. Regenstein, M.; Andres, E. Reducing hospital readmissions among medicaid patients: A review of the literature. Qual. Manag. Healthc. 2014, 23, 203-225. [CrossRef] [PubMed]

5. Purdy, S.; Griffin, T.; Salisbury, C.; Sharp, D. Ambulatory care sensitive conditions: Terminology and disease coding need to be more specific to aid policy makers and clinicians. Public Health 2009, 123, 169-173. [CrossRef]

6. Lu, S.; Kuo, D.Z. Hospital charges of potentially preventable pediatric hospitalizations. Acad. Pediatr. 2012, 12, 436-444. [CrossRef] [PubMed]

7. Kenyon, C.C.; Melvin, P.R.; Chiang, V.W.; Elliott, M.N.; Schuster, M.A.; Berry, J.G. Rehospitalization for childhood asthma: Timing, variation, and opportunities for intervention. J. Pediatr. 2014, 164, 300-305. [CrossRef]

8. Krieger, J. Home is where the triggers are: Increasing asthma control by improving the home environment. Pediatr. Allergy Immunol. Pulmonol. 2010, 23, 139-145. [CrossRef]

9. Takaro, T.K.; Krieger, J.; Song, L.; Sharify, D.; Beaudet, N. The breathe-easy home: The impact of asthma-friendly home construction on clinical outcomes and trigger exposure. Am. J. Public Health 2011, 101, 55-62. [CrossRef]

10. Howrylak, J.A.; Spanier, A.J.; Huang, B.; Peake, R.W.A.; Kellogg, M.D.; Sauers, H.; Kahn, R.S. Cotinine in children admitted for asthma and readmission. Pediatrics 2014, 133, e355-e362. [CrossRef]

11. Vicendese, D.; Dharmage, S.C.; Tang, M.L.K.; Olenko, A.; Allen, K.J.; Abramson, M.J.; Erbas, B. Bedroom air quality and vacuuming frequency are associated with repeat child asthma hospital admissions. J. Asthma 2015, 52, 727-731. [CrossRef]

12. Kim, J.J. Ambient air pollution: Health hazards to children. Pediatrics 2004, 114, 1699-1707. [CrossRef] [PubMed]

13. Cook, A.G.; Annemarie, J.B.M.; Pereira, G.; Jardine, A.; Weinstein, P. Use of a total traffic count metric to investigate the impact of roadways on asthma severity: A case-control study. Environ. Health 2011, 10, 52. [CrossRef] [PubMed]

14. Brown, M.S.; Sarnat, S.E.; DeMuth, K.A.; Brown, L.A.S.; Whitlock, D.R.; Brown, S.W.; Tolbert, P.E.; Fitzpatrick, A.M. Residential proximity to a major roadway is associated with features of asthma control in children. PLoS ONE 2012, 7, e37044. [CrossRef] [PubMed]

15. Newman, N.C.; Ryan, P.H.; Huang, B.; Beck, A.F.; Sauers, H.S.; Kahn, R.S. Traffic-related air pollution and asthma hospital readmission in children: A longitudinal cohort study. J. Pediatr. 2014, 164, 1396-1402.e1. [CrossRef] [PubMed] 
16. Delfino, R.J.; Chang, J.; Wu, J.; Ren, C.; Tjoa, T.; Nickerson, B.; Cooper, D.; Gillen, D.L. Repeated hospital encounters for asthma in children and exposure to traffic-related air pollution near the home. Ann. Allergy Asthma Immunol. 2009, 102, 138-144. [CrossRef]

17. Chang, J.; Delfino, R.J.; Gillen, D.; Tjoa, T.; Nickerson, B.; Cooper, D. Repeated respiratory hospital encounters among children with asthma and residential proximity to traffic. Occup. Environ. Med. 2009, 66, 90-98. [CrossRef]

18. Centers for Disease Control and Prevention. National Environmental Public Health Tracking Network. 2018. Available online: www.cdc.gov/ephtracking (accessed on 1 August 2019).

19. Centers for Disease Control and Prevention. Downloadable Datasets. 2018. Available online: https: //ephtracking.cdc.gov/download (accessed on 1 August 2019).

20. Centers for Disease Control and Prevention. Outdoor Air. In: Monitor + Model Air Data. 2019. Available online: https://ephtracking.cdc.gov/showAirMonModData (accessed on 1 August 2019).

21. Liu, H.; Tian, Y.; Cao, Y.; Song, J.; Huang, C.; Xiang, X.; Li, M.; Hu, Y. Fine particulate air pollution and hospital admissions and readmissions for acute myocardial infarction in 26 Chinese cities. Chemosphere 2018, 192, 282-288. [CrossRef]

22. O'Lenick, C.R.; Winquist, A.; Mulholland, J.A.; Friberg, M.D.; Chang, H.H.; Kramer, M.R.; Darrow, L.A.; Sarnat, S.E. Assessment of neighbourhood-level socioeconomic status as a modifier of air pollution-asthma associations among children in Atlanta. J. Epidemiol. Community Health 2017, 71, 129-136. [CrossRef]

23. Orellano, P.; Quaranta, N.; Reynoso, J.; Balbi, B.; Vasquez, J. Effect of outdoor air pollution on asthma exacerbations in children and adults: Systematic review and multilevel meta-analysis. PLoS ONE 2017, 12, e0174050. [CrossRef]

24. Zhang, Y.; Ni, H.; Bai, L.; Cheng, Q.; Zhang, H.; Wang, S.; Xie, M.; Zhao, D.; Su, H. The short-term association between air pollution and childhood asthma hospital admissions in urban areas of Hefei City in China: A time-series study. Environ. Res. 2019, 169, 510-516. [CrossRef]

25. Hua, J.; Yin, Y.; Peng, L.; Du, L.; Geng, F.; Zhu, L. Acute effects of black carbon and PM2.5 on children asthma admissions: A time-series study in a Chinese city. Sci. Total Environ. 2014, 481, 433-438. [CrossRef]

26. Ding, L.; Zhu, D.; Peng, D.; Zhao, Y. Air pollution and asthma attacks in children: A case-crossover analysis in the city of Chongqing, China. Environ. Pollut. 2017, 220, 348-353. [CrossRef]

27. Li, S.; Batterman, S.; Wasilevich, E.; Wahl, R.; Wirth, J.; Su, F.-C.; Mukherjee, B. Association of daily asthma emergency department visits and hospital admissions with ambient air pollutants among the pediatric Medicaid population in Detroit: Time-series and time-stratified case-crossover analyses with threshold effects. Environ. Res. 2011, 111, 1137-1147. [CrossRef] [PubMed]

28. Zu, K.; Liu, X.; Shi, L.; Tao, G.; Loftus, C.T.; Lange, S.; Goodman, J.E. Concentration-response of short-term ozone exposure and hospital admissions for asthma in Texas. Environ. Int. 2017, 104, 139-145. [CrossRef] [PubMed]

29. Silverman, R.A.; Ito, K. Age-related association of fine particles and ozone with severe acute asthma in New York City. J. Allergy Clin. Immunol. 2010, 125, 367-373.e5. [CrossRef] [PubMed]

30. Gharibi, H.; Entwistle, M.R.; Ha, S.; Gonzalez, M.; Brown, P.; Schweizer, D.; Cisneros, R. Ozone pollution and asthma emergency department visits in the Central Valley, California, USA, during June to September of 2015: A time-stratified case-crossover analysis. J. Asthma 2019, 56, 1037-1048. [CrossRef]

31. Ko, F.W.S.; Tam, W.; Wong, T.W.; Lai, C.K.W.; Wong, G.W.K.; Leung, T.F.; Ng, S.S.S.; Hui, D.S.C. Effects of air pollution on asthma hospitalization rates in different age groups in Hong Kong. Clin. Exp. Allergy 2007, 37, 1312-1319. [CrossRef]

32. Son, J.-Y.; Lee, J.-T.; Park, Y.H.; Bell, M.L. Short-term effects of air pollution on hospital admissions in Korea. Epidemiology 2013, 24, 545-554. [CrossRef]

33. Iskandar, A.; Andersen, Z.J.; Bønnelykke, K.; Ellermann, T.; Andersen, K.K.; Bisgaard, H. Coarse and fine particles but not ultrafine particles in urban air trigger hospital admission for asthma in children. Thorax 2012, 67, 252-257. [CrossRef]

34. Villeneuve, P.J.; Chen, L.; Rowe, B.H.; Coates, F. Outdoor air pollution and emergency department visits for asthma among children and adults: A case-crossover study in northern Alberta, Canada. Environ. Health 2007, 6, 40. [CrossRef] [PubMed] 
35. Strickland, M.J.; Darrow, L.A.; Klein, M.; Flanders, W.D.; Sarnat, J.A.; Waller, L.A.; Sarnat, S.E.; Mulholland, J.A.; Tolbert, P.E. Short-term associations between ambient air pollutants and pediatric asthma emergency department visits. Am. J. Respir. Crit. Care Med. 2010, 182, 307-316. [CrossRef] [PubMed]

36. Gleason, J.A.; Bielory, L.; Fagliano, J.A. Associations between ozone, PM2.5, and four pollen types on emergency department pediatric asthma events during the warm season in New Jersey: A case-crossover study. Environ. Res. 2014, 132, 421-429. [CrossRef] [PubMed]

37. Li, X.; Chen, Q.; Zheng, X.; Li, Y.; Han, M.; Liu, T.; Xiao, J.; Guo, L.; Zeng, W.; Zhang, J.; et al. Effects of ambient ozone concentrations with different averaging times on asthma exacerbations: A meta-analysis. Sci. Total Environ. 2019, 691, 549-561. [CrossRef] [PubMed]

38. Jalaludin, B.; Khalaj, B.; Sheppeard, V.; Morgan, G. Air pollution and ED visits for asthma in Australian children: A case-crossover analysis. Int. Arch. Occup. Environ. Health 2008, 81, 967-974. [CrossRef]

39. Bateson, T.F.; Schwartz, J. Children's response to air pollutants. J. Toxicol Environ. Health A 2008, 71, $238-243$. [CrossRef]

40. Diffey, B.L. An overview analysis of the time people spend outdoors. Br. J. Dermatol. 2011, 164, 848-854. [CrossRef]

41. Goldman, G.T.; Mulholland, J.A.; Russell, A.G.; Strickland, M.J.; Klein, M.; Waller, L.A.; Tolbert, P.E. Impact of exposure measurement error in air pollution epidemiology: Effect of error type in time-series studies. Environ. Health 2011, 10, 61. [CrossRef]

42. Parikh, K.; Hall, M.; Kenyon, C.C.; Teufel, R.J.; Mussman, G.M.; Montalbano, A.; Gold, J.; Antoon, J.W.; Subramony, A.; Mittal, V.; et al. Impact of discharge components on readmission rates for children hospitalized with asthma. J. Pediatr. 2018, 195, 175-181.e2. [CrossRef]

43. Kash, B.A.; Baek, J.; Cheon, O.; Coleman, N.E.; Jones, S.L. Successful hospital readmission reduction initiatives: Top five strategies to consider implementing today. Int. J. Hosp. Tour. Adm. 2018, 7, 16-23. [CrossRef]

44. Baek, J.; Huang, K.; Conner, L.; Tapangan, N.; Xu, X.; Carrillo, G. Effects of the home-based educational intervention on health outcomes among primarily Hispanic children with asthma: A quasi-experimental study. BMC Public Health 2019, 19, 912. [CrossRef] [PubMed]

45. Carrillo, G.; Spence-Almaguer, E.; Lucio, R.L.; Chong-Menard, B.; Smith, K. Improving asthma in Hispanic families through a home-based educational intervention. Pediatr. Allergy Immunol. Pulmonol. 2015, 28, 165-171. [CrossRef]

46. Fernandes, J.C.; Biskupiak, W.W.; Brokaw, S.M.; Carpenedo, D.; Loveland, K.M.; Tysk, S.; Volg, S. Outcomes of the Montana Asthma Home Visiting Program: A home-based asthma education program. J. Asthma 2019, 56, 104-110. [CrossRef] [PubMed]

47. Kash, B.A.; Baek, J.; Davis, E.; Champagne-Langabeer, T.; Langabeer II, J.R. Review of successful hospital readmission reduction strategies and the role of health information exchange. Int. J. Med. Inform. 2017, 104, 97-104. [CrossRef]

(C) 2020 by the authors. Licensee MDPI, Basel, Switzerland. This article is an open access article distributed under the terms and conditions of the Creative Commons Attribution (CC BY) license (http://creativecommons.org/licenses/by/4.0/). 